\title{
多齿络合法分离锂同位素的冠醚化学
}

\author{
刘华 $a$ 黄祚刚*,a 文 珂*,a 姜 标*,a,b \\ ( ${ }^{a}$ 中国科学院上海高等研究院 上海 201210) \\ $\left({ }^{b}\right.$ 中国科学院上海有机化学研究所 上海 200032)
}

\begin{abstract}
摘要 锂的两种天然同位素锂-6 和锂-7 在核能工业中具有重要的作用. 在众多锂同位素的分离方法和体系中, 多齿络 合法普遍具有较高的同位素分离系数. 冠醚由于其对金属阳离子具有较强的选择性络合能力，被认为是多齿络合法分 离锂同位素最有效的萃取剂. 综述了冠醚及冠醚树脂在多齿络合法分离锂同位素中的应用, 并简述新型金属冠醚与金 属离子络合的进展，展望其在锂同位素分离中的潜在应用前景.
\end{abstract}

关键词＼cjkstart锂同位素分离; 多齿络合法; 冠醚; 冠醚树脂; 金属杂冠梄

\section{Crown Ether Chemistry of Polydentate Complexing for Lithium Isotope Separation}

\author{
Liu, Hua ${ }^{a} \quad$ Huang, Zuogang ${ }^{*, a} \quad$ Wen, Ke $e^{*, a} \quad$ Jiang, Biao ${ }^{*, a, b}$ \\ ( ${ }^{a}$ Shanghai Advanced Research Institute, Chinese Academy of Sciences, Shanghai 201210) \\ ( ${ }^{b}$ Shanghai Institute of Organic Chemistry, Chinese Academy of Sciences, Shanghai 200032)
}

\begin{abstract}
The two naturally occurring isotopes of lithium, ${ }^{6} \mathrm{Li}$ and ${ }^{7} \mathrm{Li}$, have important roles in the nuclear energy industry. Among many techniques for the separation of lithium isotopes, high isotope separation factors are usually realized with the polydentate complex method. Crown ethers, for their selective-complexing ability with metal cations, have been regarded as perhaps the most effective extractants for lithium isotope separation. This review summarizes the application of crown ether and crown ether chelating resin for lithium isotope separation based on polydentate complexing method, exemplify recent developments in complexation of metal-containing crown ethers with metal cations, and prospect their potential in separation of lithium isotopes.
\end{abstract}

Keywords lithium isotope separation; polydentate complex; crown ether; crown ether chelating resin; metal-containing crown ethers

在核能工业中, 锂的两种同位素锂- 6 和锂-7 具有不 同的核反应性能. 锂-6 受热中子轰击后产生的気( $\mathrm{T}$ ), 是 未来可控热核聚变反应堆必不可少的核聚变燃料. 锂-7 在裂变反应堆中具有重要用途, 氢氧化锂 $\left({ }^{7} \mathrm{LiOH}\right)$ 可用 于控制压水堆 $\mathrm{pH}$ 值, 减缓压力容器壁的腐蚀; 氟化锂 ( $\left.{ }^{7} \mathrm{LiF}\right)$ 可作为新一代熔融盐反应堆(MSR)冷却剂的重要 组分, 提高反应堆的安全性和效率 ${ }^{[1]}$.

锂同位素的分离方法很多, 如化学交换法(锂录齐 法)、离子交换法、电迁移/熔岩电解法、热扩散/分子蒸 馏法、激光/电磁分离法、以及萃取、分级结晶、分级沉 淀等 ${ }^{[2 \sim 4]}$. 其中已用于工业生产的只有锂录齐法 ${ }^{[5]}$, 但 此工艺大量的使用金属录, 严重危害人体和环境安全 ${ }^{[6]}$,
因而无录分离锂同位素的工艺研发具有重要的意义, 吸 引了持续的关注.

自从 1960 年 Pedersen ${ }^{[7]}$, Lehn 等 ${ }^{[8]}$ 分别报道了冠醚 和穴醚以来，这种大环醚类对金属阳离子的高度选择性 络合作用得到了广泛的研究 ${ }^{[9-13]}$, 基于大环醚类的络合 分离法分离锂同位素的技术随之被广泛研究.

陈耀焕 ${ }^{[15]}$ 、李廷伍 ${ }^{[16]}$ 和顾志国等 ${ }^{[17]}$ 分别从不同角度 综述了锂同位素的分离进展. 考虑到冠醚化学的快速发 展及其在锂同位素分离中的潜在工业应用前景, 本文着 重基于冠醚化学在多齿络合法分离锂同位素中的应用 进行总结, 主要包括冠醚单体作为萃取剂, 在液-液萃 取法中的应用; 冠醚作为树脂活性单元，在离子交换色

*E-mail: jiangb@sari.ac.cn

Received July 19, 2013; revised October 11, 2013; published online October 16, 2013.

Project supported by the "Stategic Priority Research Program" of the Chinese Academy of Science (No. XDA01020304).

中国科学院战略性先导科技专项(No. XDA01020304)资助项目. 
谱法中的应用; 并且结合近年来新型金属辅助的冠醚在 与金属离子选择性络合方面的进展, 展望其在锂同位素 分离中的潜在应用前景.

\section{1 冠醚单体作为萃取剂在液一液萃取法分离锂 同位素中的应用}

传统的络合萃取法为液-液两相(有机相和水相)分 离体系, 通常包括萃取、交换、反萃三个环节. 通过该 法实现锂同位素分离的关键在于选择合适的萃取体系， 即特定的萃取剂、协萃剂和稀释剂. 早在 1930 年代就有 溶剂萃取法分离锂同位素的报道, 但从目前公开资料 看, 该类体系并未取得较好的分离效果 ${ }^{[15]}$.

1979 年, Jepson 等 ${ }^{[14]}$ 首次报道了使用穴醚 $(2,2,1)$ (1)分离锂同位素的方法, 其分离系数高达 1.041(锂同位 素分离的理论分离系数为 1.08). 穴醚之所以表现出很 强的络合能力和高度的选择性, 是因为它具有三维结 构, 能较好的包容金属离子, 并且参与配位的氧原子和 氮原子在空间分布均匀, 有利于其与金属离子的配位络 合.

1982 年, 支克正等 ${ }^{[18]}$ 验证了穴醚对锂同位素的络 合分离效应, 并考察了不同空穴尺寸和不同配位原子数 目的穴醚 $(2,2,1)(\mathbf{1})$ 、穴醚 $(2,1,1)(\mathbf{2})$ 和穴醚 $(2,2,2)(3)$ 对锂同位素分离的效果, 3 种穴醚在不同的萃取体系中 均都能取得较好的分离效果, 其分离系数大小顺序为: $\mathbf{1}>\mathbf{2}>\mathbf{3}$, 即空穴尺寸稍大于锂离子直径的穴醚 $(2,2$,

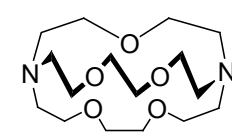

1

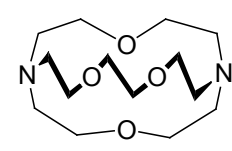

2

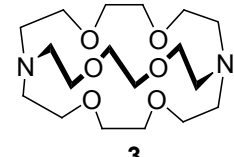

3

图 1 穴醚 1,2 和 3 的结构

Figure 1 Structures of cryptands 1, 2 and 3

1)体现出了最好的锂同位素分离效应.

考虑到单环结构的冠醚通常较容易合成且价格相 对便宜, 1983 年, 支克正课题组 ${ }^{[19]}$ 报道了 7 种不同的单 环冠醚萃取体系, 包括 15-冠-5 及其衍生物 $(\mathbf{4} \sim 6)$ 、饱和 的八甲基四氧 quaterene (7)和开链冠醚 $(8 \sim 10)$, 测定了 各体系中锂同位素交换反应速度, 分离系数及锂在两相 中的分配比. 各体系的锂同位素分离系数在 1.012 1.030 之间, 其中最高的为环已基-15-冠-5 (5)体系. 文中 还就冠醚结构, 包括空穴大小、冠梄类型及其所带有的 边环及边环上的取代基, 对同位素分离系数和锂在两相 中的分配的影响提出了初步的看法, 认为: 冠醚的空间 尺寸应稍大于锂离子的直径; 选择合适的边环及边环取 代基可以大大减小其水溶性，增大其在有机相中的溶解 度, 从而提高对锂的萃取能力.

1984 年, 方胜强等 ${ }^{[20]}$ 报道了 $4,4^{\prime}$-二叔丁基二苯 并-30-冠-10 (11)与硫氰酸锂络合过程中的锂同位素分 离效应, 其同位素单级分离系数可达 1.036 , 该体系交 换速度快, 反萃容易. 1987 年, 该课题组 ${ }^{[21]}$ 又考察了 4叔<smiles>C1COCCOCCOCCOCCO1</smiles>

4

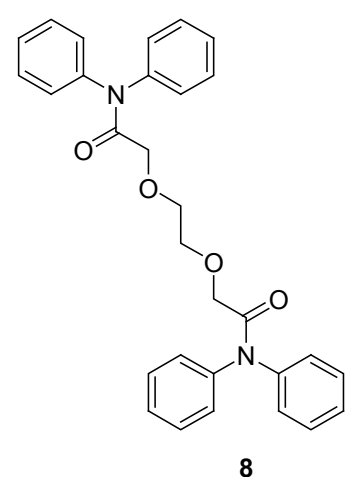<smiles>CC(C)(C)C1CCC2OCCOCCOCCOCC2O1</smiles>

6

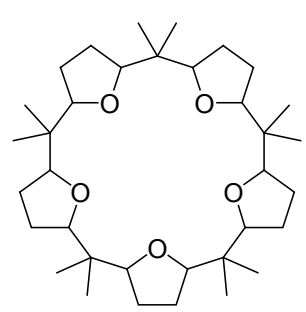

7<smiles>O=C(COCCOCC(=O)N(Cc1ccccc1)Cc1ccccc1)N(Cc1ccccc1)Cc1ccccc1</smiles><smiles>C/C=C\C=C/C</smiles>

10

图 2 冠醚及类似物 $4 \sim 10$ 的结构

Figure 2 Structures of crown ethers and analogues $\mathbf{4} \sim \mathbf{1 0}$ 
丁基苯并-15-冠-5 (12)对锂盐的同位素分离效应, 测得 其同位素分离系数为 1.035 .

1985 年, 陈耀焕等 ${ }^{[22]}$ 根据 4 类锂的离子对萃取原理 (图 3)研究溶剂萃取分离锂同位素中的阴离子结构效应, 发现对于环醚隔离离子对体系，降低阴离子的电荷密度 和硬度, 有利于萃取分配比和同位素分离系数的提高.

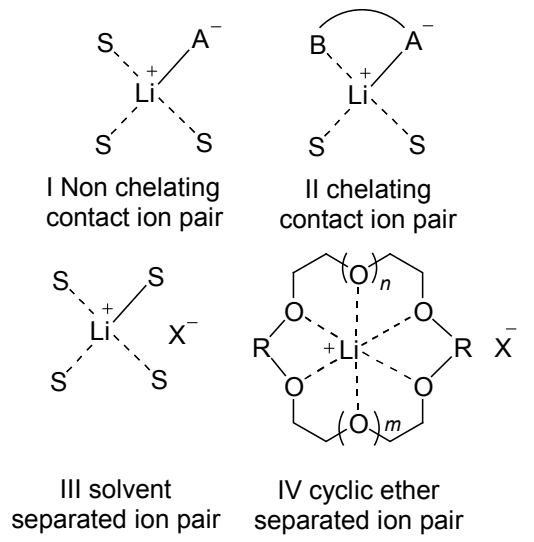

图 3 四种锂的离子对萃取体系

Figure 3 Four kinds of ion-pair extraction systems of lithium

1986 年, 姜延林等 ${ }^{[23]}$ 报道研究了 8 种锂盐-冠醚络 合物化学交换体系, 发现了同位素单级分离系数高达 1.042 的 4-甲基-苯并-15-冠-5 (13)(图 4)体系, 并且采用 三氯乙酸根代替 Jepson 等 ${ }^{[14]}$ 使用的三氟乙酸根离子, 可 大大减低成本. 文中还指出: 锂离子在水中与水结合成 水合离子，而大环多醚的环形和球型结构能够全部或大 部分排除与锂离子强烈缔合的水分子，两相化合物的对 称伸缩振动频率变化也较大, 故而有利于同位素分离系 数的提高.

1989 年, 方胜强等 ${ }^{[24]}$ 考察了多梄液-液萃取体系中 冠醚的内结构、侧基, 冠醚浓度, 有机溶剂, 锂盐阴离 子，锂盐浓度等因素对锂的热力学同位素效应的影响. 随后，该课题组 ${ }^{[25]}$ 报道了使用 4-甲基-苯并-15-冠-5 (13) 萃取硫氧酸锂的锂同位素分离能力, 同位素分离系数可 达 1.039 , 并且在高浓度下仍保持较高的同位素分离能 力; 而双环冠醚对甲苯氧基联苯桥联双苯并-15-冠-5 (14) 萃取硫氰酸锂的的锂同位素分离能力较相应单环 冠醚小 ${ }^{[26]} .1994$ 年, 该课题组 ${ }^{[27]}$ 通过对冠醚萃取体系的 混合锂盐效应的研究发现适当地选用混合锂盐料液可 以获得更大的锂同位素分离系数. 例如, 用 $0.78 \mathrm{~mol} / \mathrm{L}$ 的 $12-\mathrm{C}_{6} \mathrm{H}_{5} \mathrm{I}$ 溶液, 按相比 $1: 1$, 分别萃取 $3.25 \mathrm{~mol} / \mathrm{L}$ 的 $\mathrm{LiClO}_{4}$ 和 $4.06 \mathrm{~mol} / \mathrm{L}$ 混盐 $\left(\mathrm{LiClO}_{4}+\mathrm{Li}-\mathrm{EDTA}\right)$ 中的锂, 所 得锂同位素分离系数分别为 1.027 和 1.033 .

1999 年, 金建南等 ${ }^{[28]}$ 研究了酰胺型开链冠醚 PAIV- $40 \%$ 正辛醇-60\%磺化煤油体系，在不同酸度下对 锂盐的萃取性能, 取得 $1.021 \sim 1.033$ 的锂同位素分离<smiles>Cc1cc(C(C)(C)C)ccc1OCCOCCOCCOCCOc1ccc(C(C)(C)C)cc1OCCOCCOCCOCCOCCO</smiles>

11

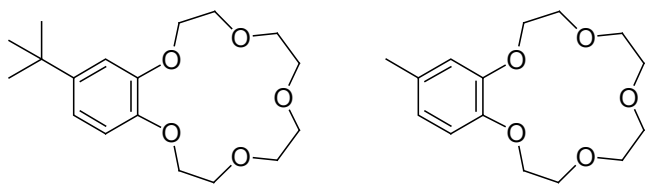

12

13

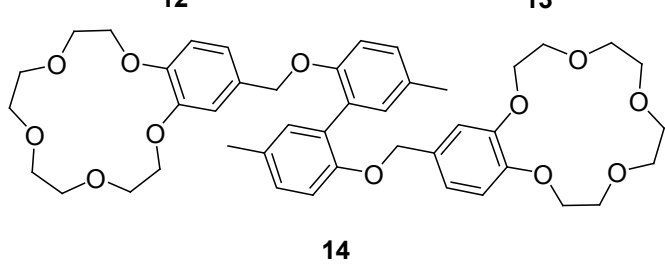

图 4 冠醚 11 14 的结构

Figure 4 Structures of crown ethers 11 $\sim 14$

系数. 这类开链冠醚易合成, 产率高, 对锂的萃取能力 与冠醚相当，若对其进行适当的结构修饰，将具有实用 前景.

冠醚类化合物作为液-液萃取法分离锂同位素中的 有效协萃剂, 得到了较为深入的研究和探讨, 这些体系 普遍具有很好的单级分离效应，但考虑到其需要多级相 分离，浓缩，相转换操作，并且冠醚合成较复杂，该体 系在实际应用中还存在一定的局限性，有待于进一步发 展成熟.

\section{2 冠梄作为树脂活性单元在离子交换色谱法分 离锂同位素中的应用}

早在 1930 年代, 离子交换色谱法即被用来尝试分 离锂同位素，随后的几十年中，该法也得到了较广泛的 研究和并获得了快速的发展 ${ }^{[17]}$. 冠醚类化合物在液液 萃取法分离锂同位素中表现出来的较高分离效应，为离 子色谱法提供了新的途径，冠醚树脂在离子交换色谱法 分离锂同位素的应用研究随之展开(注：以下冠醚结构 式中 MR 表示梅氏树脂, MPR 表示梅氏多肽树脂).

1983 年, Fujine 等 ${ }^{[29]}$ 首先使用商品化的穴醚 $\left(2_{\mathrm{B}}, 2,1\right)$ 聚合物(Merck: Kryptofix 221B 聚合物)树脂 15 分离锂同 位素，锂-7 富集于色谱柱的前端，而锂-6 富集于色谱柱 的后端, 得到 1.014 的分离系数. 随后 Nishizawa 等 ${ }^{[30]}$ 使用相同的冠醚树脂富集卤化锂甲醇溶液中的锂同位 素, 得到同位素单级分离系数最高达 1.047 . 


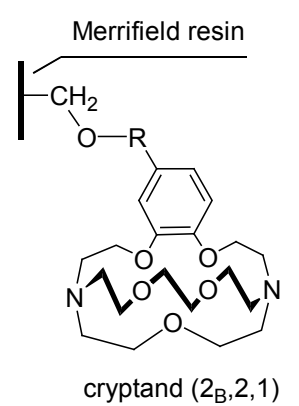

15

图 5 树脂 15 的结构

Figure 5 Structure of resin $\mathbf{1 5}$

1991 年以来, Kim 课题组 ${ }^{[31]}$ 将各种特定结构的冠醚 负载于树脂上, 考察其分离锂同位素的能力. 将 4-氨基 苯并-15-冠-5 与氯甲基化的苯乙烯-DVB 共聚物接连合 成树脂 16 (Scheme 1), 考察其对不同溶剂、不同阴离子 的锂盐的锂同位素分离效应, 据称同位素单级分离系数 可达 1.053 .

1995 年, Kim 课题组 ${ }^{[32]}$ 将氮杂冠醚 DBPDA 和 RDBPDA 与梅氏树脂连接成树脂 17 和 18 (Scheme 2), 在相同溶剂中, 两种树脂的同位素分离能力相当, 例如 在乙腈中同位素分离系数分别高达 1.034 和 1.035 , 两种 树脂的同位素分离能力与所用溶剂密切相关, 溶剂介电 常数增大不利于锂同位素的分离.

1997 年, Kim 课题组 ${ }^{[33]}$ 合成了间三氮杂-18-冠-6 冠 醚, 并将其与梅氏树脂接连成树脂 19 (Scheme 3), 其分
离锂同位素的分离系数可高达 1.068 .

采用类似的合成方法，该课题组 ${ }^{[34]}$ 合成了 $\mathrm{N}_{4} \mathrm{O}_{2}$ 氮 杂冠醚树脂 $20, \mathrm{~N}_{4} \mathrm{O}_{2}$ 枝接量为 $3.8 \mathrm{meq} / \mathrm{g}$, 可得 1.038 的 同位素单级分离系数; $\mathrm{N}_{4} \mathrm{~S}_{2}$ 硫氮杂冠醚树脂 $21, \mathrm{~N}_{4} \mathrm{~S}_{2}$ 枝 接量为 $0.34 \mathrm{meq} / \mathrm{g}$, 可得 1.034 的同位素单级分离系 数 ${ }^{[35]}$; 对于 $\mathrm{N}_{4} \mathrm{O}$ 氮杂冠醚树脂 $2^{[36]}$ 以及 $\mathrm{N}_{3} \mathrm{O}_{2}$ 氮杂冠醚 树脂 $\mathbf{2 3}^{[37]}$, 则分别只有 1.00127 及 1.00104 的同位素分 离系数.

2002 年, $\mathrm{Kim}$ 课题组 ${ }^{[38]}$ 报道了另一种结构的 $\mathrm{N}_{3} \mathrm{O}_{3}$ 氮杂冠醚树脂 24, 该体系得到 1.028 的单级分离系数; 而对于三氮杂冠醚 TOTA 树脂 $\mathbf{2 5}$, 其同位素单级分离系 数为 $1.027^{[39]}$.

此外, Kim 课题组合成并考察了化合物 NOTE 分离 锂同位素的效应，同位素分离系数为 $1.024^{[40]}$; 将 NOTE 枝接成树脂 26 后, 其同位素分离系数为 $1.026^{[41]}$; 对于 同类 NDOE 树脂 27 , 其同位素分离系数为 $1.0201^{[42]}$. 该 课题组还将 4-氨基苯并-15-冠-5 (AB15C5)枝接成树脂 28 , 枝接量为 $0.24 \mathrm{meq} / \mathrm{g}$, 测得锂同位素的单级分离系 数为 $1.026^{[43]}$. 2003 年, 该课题组用相同的方法合成了 4-氨基苯并-18-冠-6 (AB18C6)树脂 29, 枝接量为 2.25 $\mathrm{meq} / \mathrm{g}$, 测得锂同位素的单级分离系数为 $1.0095^{[44]}$. 对 比可知，树脂中活性单元结构的空间大小对锂同位素的 分离效果具有较大影响.

2002 年, Fujii 等 ${ }^{[45]}$ 考虑到通常合成所得的冠醚树

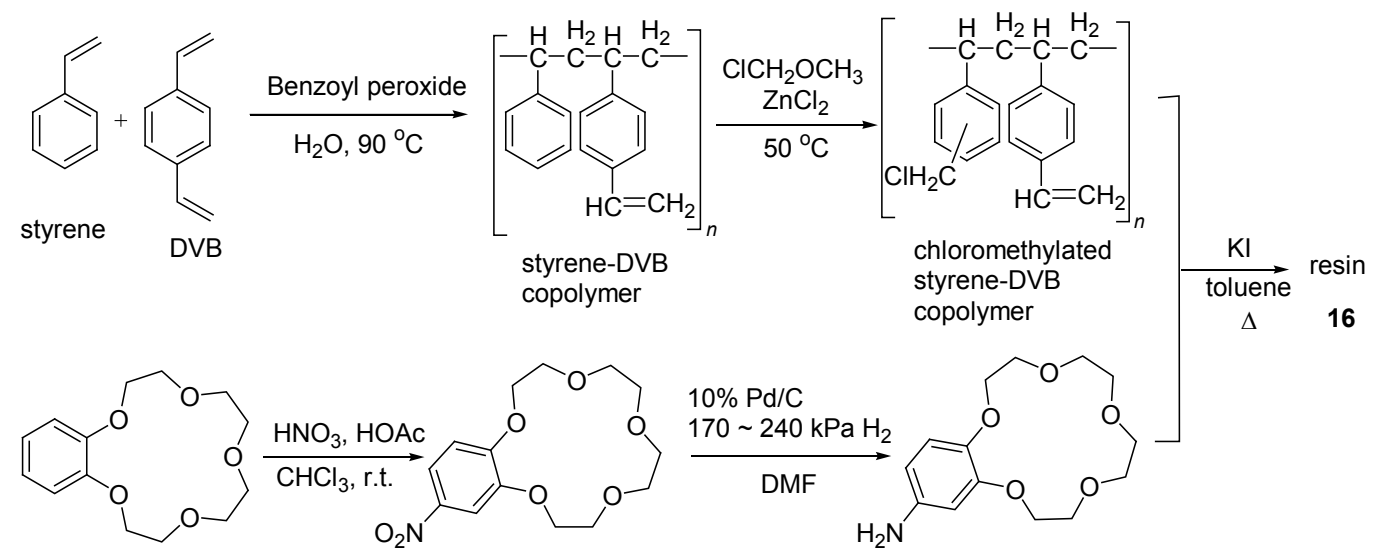

Scheme 1

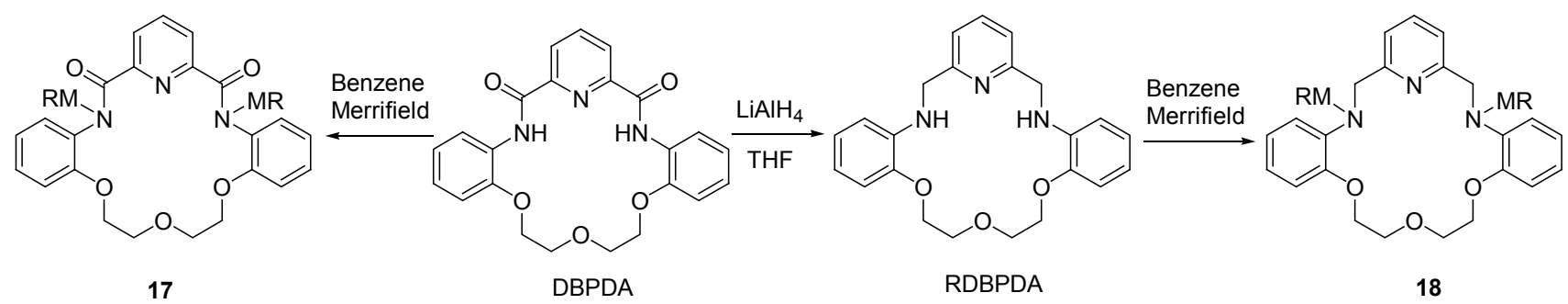

Scheme 2 

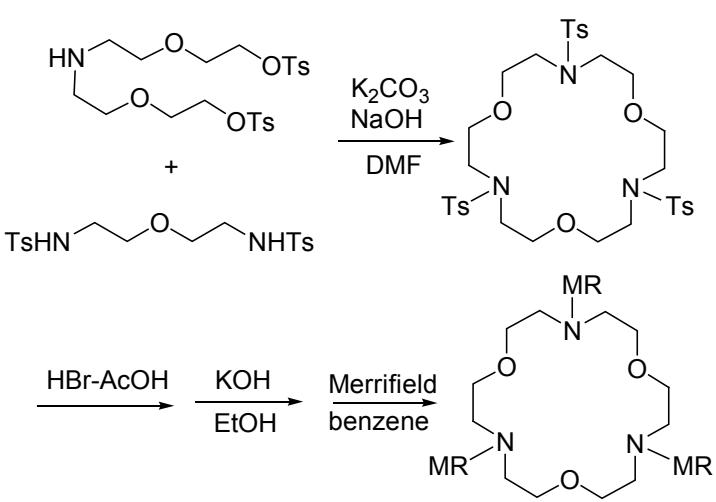

19

Scheme 3

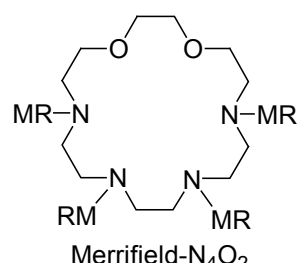

20

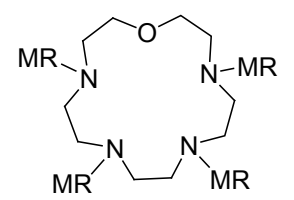

Merrifield- $\mathrm{N}_{4} \mathrm{O}$

22

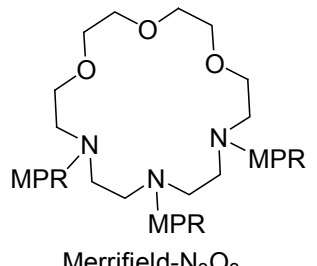

24

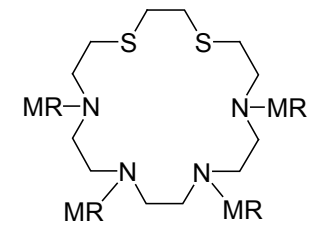

Merrifield- $\mathrm{N}_{4} \mathrm{~S}_{2}$

21

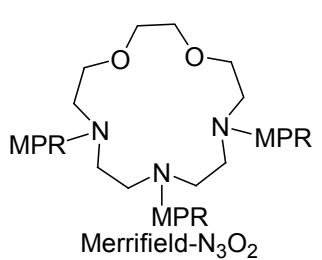

23

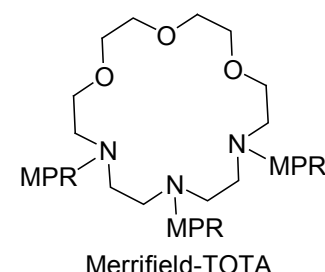

25

图 6 树脂 $20 \sim 25$ 的结构

Figure 6 Structures of resins $\mathbf{2 0} \sim \mathbf{2 5}$

脂颗粒大小不一, 使得柱层析分离锂同位素的效果有所 下降，故使用具有特定尺寸的多孔硅胶与苯并-15-冠-5 (B15C5)枝接, 得到酚醛树脂化 B15C5 冠醚 $\mathbf{3 0}$, 测得其 分离锂同位素的分离系数为 1.0127. 2006 年, 该课题 组 ${ }^{[46]}$ 又报道了一种新方法制备的酚醛树脂化 $\mathrm{B} 15 \mathrm{C} 5$ 冠 醚, 其同位素分离系数为 1.033. 2007 年, Jeon 等 ${ }^{\left[{ }^{[7]}\right.}$ 合成 了两种四氮杂大环化合物树脂 $\mathbf{3 1}$ 和 $\mathbf{3 2}$, 枝接量分别为 3.5 和 $1.71 \mathrm{meq} / \mathrm{g}$, 但所测锂同位素分离系数分别只有 1.0005204 和 1.0001246 . 作者认为二者锂同位素分离系 数的差别主要是由单体枝接量的差别导致的.

总体而言, 以冠醚树脂为载体的阳离子交换树脂法 分离锂同位素，同位素分离系数普遍较好，操作简便， 并且通过固-液两相分离有可能实现连续操作, 从而便

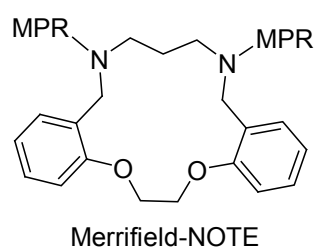

26

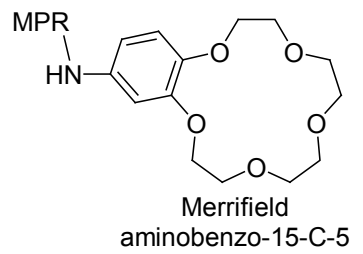

28

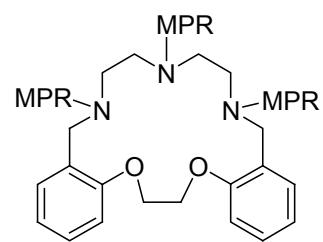

Merrifield-NDOE

27

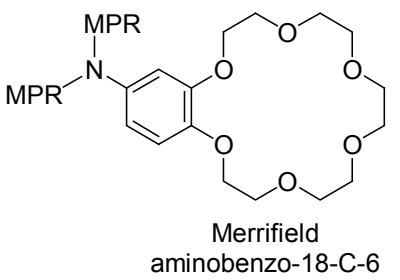

29
图 7 树脂 $26 \sim 29$ 的结构

Figure 7 Structures of resins $\mathbf{2 6} \sim \mathbf{2 9}$

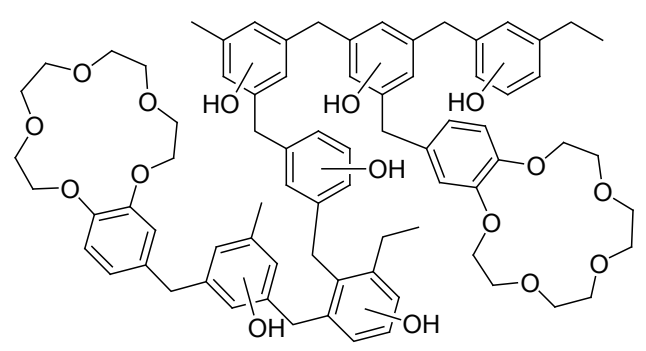

30
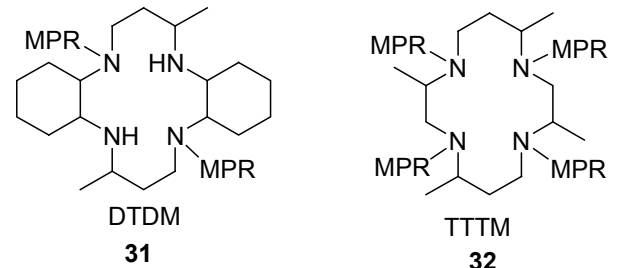

32

图 8 树脂 $30 \sim 32$ 的结构

Figure 8 Structures of resins $\mathbf{3 0} \sim \mathbf{3 2}$

于实际应用. 在上述锂同位素分离体系中，对于冠醚树 脂 16 21，27，30 32 体系，锂-6 富集于树脂相，锂-7富 集于溶剂相; 而对于冠醚树脂 $\mathbf{2 2} \sim \mathbf{2 6}, \mathbf{2 8} \sim 29$ 体系, 锂7 富集于树脂相, 锂-6 富集于溶剂相. 这可能取决于树 脂中活性单元的空间结构以及锂同位素与树脂的络合 能力较其与水络合能力的对比 ${ }^{[48]}$. 此外, 冠醚树脂中可 能仍然含有一个或多个游离的氨基, 其对锂同位素分离 能力的影响目前并没有文献报道, 其冠醚结构与分离性 能的规律和工艺条件仍有待进一步研究.

\section{3 新型冠醚类似物在锂同位素分离中的潜在应} 用前景

以冠醚作为萃取剂或者以冠醚作为树脂活性单元 
应用于锂同位素的分离中, 其体系普遍能够获得较高的 同位素分离系数. 但是由于冠醚合成较复杂，萃取及反 萃率偏低, 冠醚活性单体枝接量低, 吸附洗脱效率低等 各种原因, 在一定程度上限制了传统冠醚在锂同位素分 离工业中的实际应用. 要突破这种局限性, 寻找新型冠 醚及冠醚萃取体系是锂同位素分离的新思路和发展方 向.

金属杂冠醚 ${ }^{[49]}$ 作为冠醚的类似物，显示出了众多 的优越性, 例如合成简单高效, 结构刚性, 更有利于其 与金属离子的选择性络合. 其中, 金属杂 12-冠-3 系列

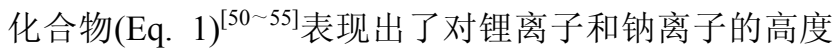
选择性络合. 通常而言, 改变桥联配体、金属离子及其 $\pi$ 配体, 不仅可以调节金属杂冠醚在溶剂中的溶解性, 更 重要的是可以调控其空腔大小, 从而实现对不同体积的 金属离子的选择性络合. Severin 等 ${ }^{[53,54]}$ 实现了通过调节 $\mathrm{pH}$ 值, 在水溶液中同步组装冠醚及络合碱金属离子, 该金属冠醚与锂离子有较大的络合常数, 并且对锂、钠 离子的选择性高达 $10000 ： 1$.

在此基础上, Severin 课题组 ${ }^{[55]}$ 还考察了三种金属杂 12-冠-3 对锂同位素的络合选择性, 其中化合物 33a 和 33b 对锂同位素没有选择性, 而 33c 则表现出了对锂-7 的络合选择性(Eq. 2). 究其原因, 是由于 33c 中与金属
$\mathrm{Ru}$ 络合的 $\pi$ 配体六甲基苯的位阻有效的阻碍了体积相 对较大的锂-6 正离子与金属杂冠醚中氧原子的络合.

基于 Severin 课题组的研究工作，Katsuta 等 ${ }^{[56]}$ 考查 了两个金属杂 12-冠-3 化合物 34 和 35(图 9)通过液液萃 取法分离碱金属离子的效果，其选择的参照物是文献报 道中对锂离子选择性最好的双苯并 14-冠 4. 研究发现, 金属杂冠醚萃取碱金属离子的平衡时间较长，并且其金 属 $\pi$ 配体位阻越大，所需平衡时间越长; 二者对不同碱 金属离子的络合常数大小顺序为: $\mathrm{Li}^{+}>\mathrm{Na}^{+}>\mathrm{K}^{+}$, 这与 离子直径大小是相匹配的; 带有 $\mathrm{Cp}$ *结构的化合物 35 对锂离子的络合能力及选择性均强于双苯并 14-冠 4 . 这些研究结果将为金属杂冠醚在液液萃取法萃取锂同 位素中的应用提供有力的参考依据.

另一类金属辅助的冠醚与碱金属离子的选择性络 合也得到了较为广泛的研究 ${ }^{[57]}$. 这类金属辅助冠醚是 在链状冠醚的末端连接螯合基团，例如二酮、水杨酸、 邻苯二酚等配位单元，在金属作用下形成环状冠醚，从 而实现其对金属离子的选择性络合(Scheme 4). 金属辅 助的冠醚普遍比相应的传统冠醚具有更高的离子络合 选择性.

值得一提的是，在含冠醚结构的分子中接入金属螯 合单元，在金属作用下可以形成新型金属螯合驱动的分
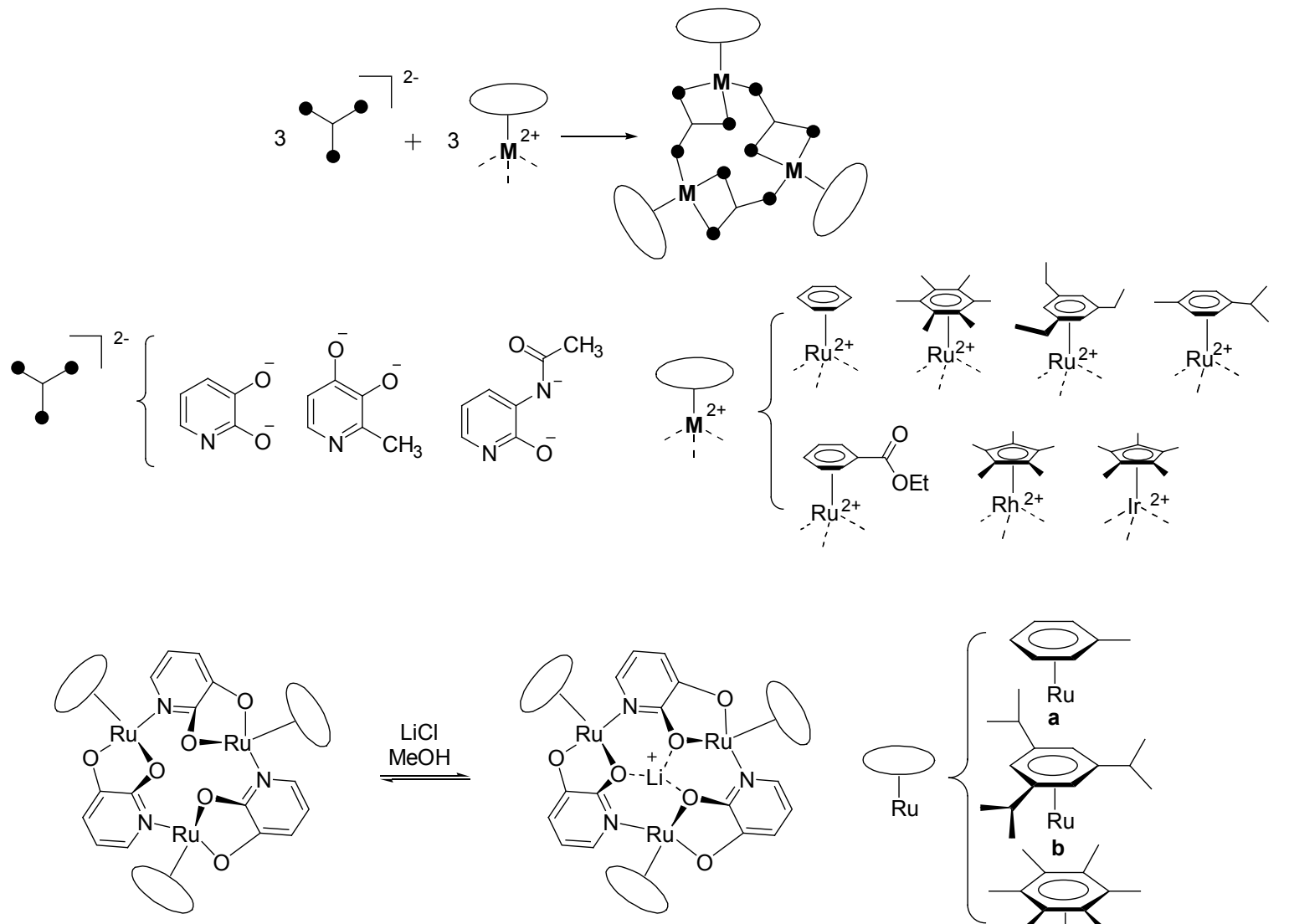

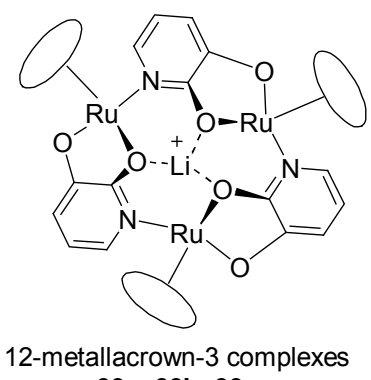

33a, 33b, 33c

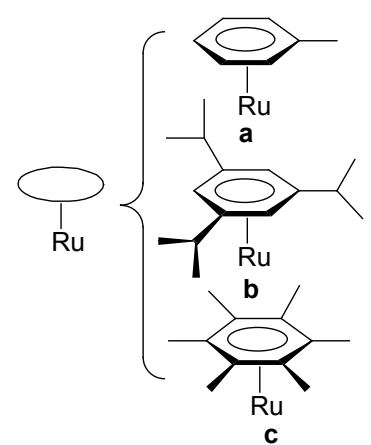

http://sioc-journal.cn/ 


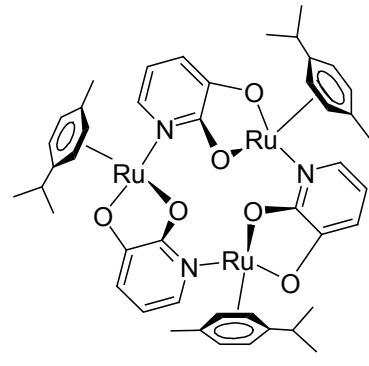

34

$\left[(p \text {-cymene }) \mathrm{Ru}\left(\mathrm{pyO}_{2}\right)\right]_{3}$

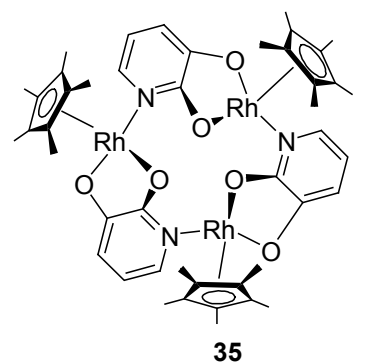

$\left[\mathrm{Cp} * \mathrm{Rh}\left(\mathrm{pyO}_{2}\right)\right]_{3}$
图 9 金属杂冠醚 $\mathbf{3 4}$ 和 $\mathbf{3 5}$ 的结构 ${ }^{[56]}$

Figure 9 Structure of metallacrown ethers $\mathbf{3 4}$ and $\mathbf{3 5}{ }^{[56]}$<smiles>COC(=O)c1cccc(OCCOCCOCCOc2cccc(C(=O)OC)c2OCCOCCOCCOc2cccc3c2O[Si](OC)O[C@H]3OC)c1O</smiles>

36<smiles>Oc1cccc(COCCOCCOCCOCc2c(O)cccc2O)c1O</smiles><smiles></smiles>

37

子钳(Scheme 5), 其具有传统冠醚对金属离子式可以为 锂同位素的选择性络合分离提供新的思路.

鉴于抗衡离子对冠醚与金属离子的络合会产生较 大的干扰，能够同时络合阴阳离子对的双功能配位受的 络合特性，同时还具有可调控的运动模式，方便金属离 子的解离，而芳香荧光基团的引入则更有利于金属络合 的检测 ${ }^{[58]}$. 这种可调控的金属络合解离模体分子亦是 配位化学研究的一个趋势, 例如 Jurkschat 等 ${ }^{[59,60]}$ 合成的 有机锡冠醚 41 (Eq. 3). 该金属冠醚的最大特征在于可 同时络合金属离子及其阴离子，从而消除了抗衡离子对 配位的影响. 此外, 化合物与 $\mathrm{LiCl}$ 的络合单晶结构显示 没有水分子的存在，这与传统冠醚络合锂离子的单晶结 构不一样. 通常而言，锂离子被水分子包合后，将不利 于它的选择性分离. 因此, 可以推测, 这种双功能配位 受体分子与锂离子的络合模式给锂离子乃至锂同位素 的选择性分离提供了新的思考方向.

此外, 2012 年, Ghosh 等 ${ }^{[61]}$ 通过理论计算考查了冠 醚-锂络合体系的各种结构, 能量及热力学平衡参数, 包括各种取代的苯并 12-冠 4 与锂离子的键能大小顺序, 并指出硝基苯较四氯化碳及氯仿更适合作为液液萃取 氯化锂的有机相. 这一理论计算结果在一定程度上为锂 同位素分离萃取提供了参考依据.

新型金属冠醚类似物通常合成简单，对金属离子的 络合选择性好，具有独特的，可调控的络合解离模式， 加之第一例金属杂冠醚对锂同位素选择性络合报道, 为 多齿络合法分离锂同位素提供了新的思路和发展方向. 新型冠醚类似物与金属离子的选择性络合研究日益广 泛和深入, 将为其在锂同位素分离中的应用提供坚实的 理论实践依据.

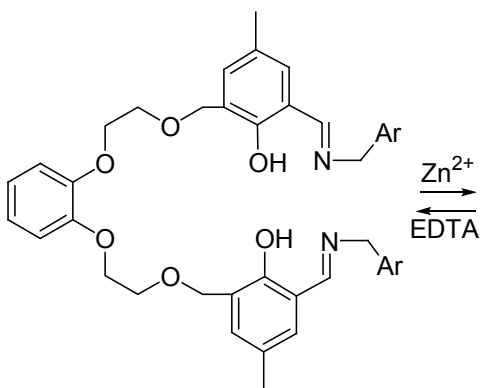

38

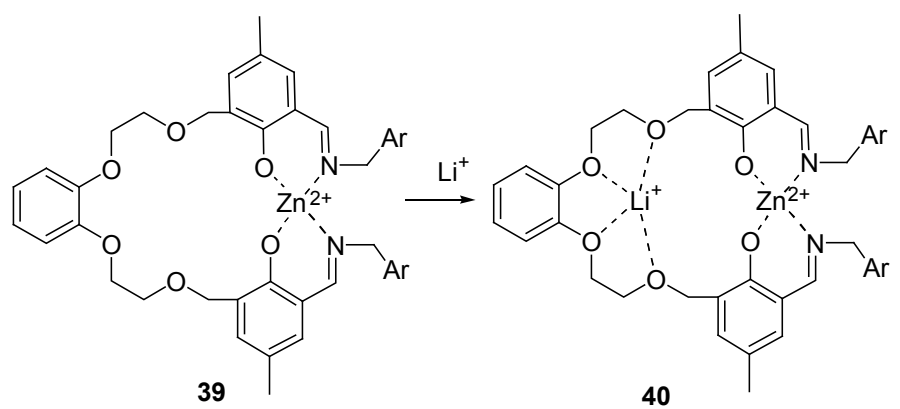

Scheme 5

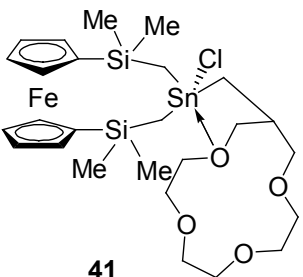<smiles>N#CC(=O)Cl</smiles>

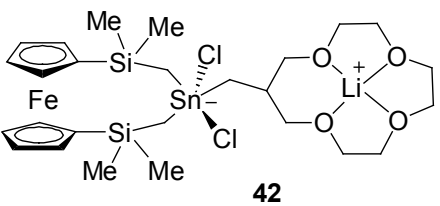




\section{4 结论与展望}

冠醚, 由于其独特的大环结构及与金属离子的选择 性络合能力, 在多齿络合法分离锂同位素中的应用得到 了较为深入的研究. 基于冠醚结构的多齿络合法普遍具 有较高的同位素分离系数, 但冠醚合成复杂、价格昂贵, 难以实现工业化应用, 发展新型冠醚及冠醚体系将是未 来的研究方向. 新型金属冠醚类似物, 由于其合成简单 高效、结构刚性、空间可调性大、与金属离子的络合选 择性高等优点, 成为了近年来主客体化学领域新的研究 热点, 加之第一例自组装的金属杂冠醚作为选择性锂同 位素受体的研究报道, 为锂同位素的分离提供了新的思 路和方向. 近年来, 冠醚及冠醚树脂在锂同位素分离中 的相关应用研究少有报道, 而新型冠醚类似物对金属离 子的选择性络合相关研究则较多, 其中不乏针对锂离子 的选择性络合研究. 随着此类研究日益广泛和深入, 将 对日后冠醚体系在锂同位素分离中的应用提供极具指 导性的理论实践基础.

\section{References}

[1] Ache, H. J. Angew. Chem., Int. Ed. 1989, 28, 1.

[2] Symons, E. A. Sep. Sci. Technol. 1985, 20, 633.

[3] Liu, Y.-F.; Fu, K.-J. At. Energy Sci. Technol. 1963, 796 (in Chinese). (刘元芳, 傅克坚, 原子能科学技术, 1963, 796.)

[4] Xiao, X.-A.; Wang, D.-X. J. Nucl. Radiochem. 1991, 13, 1 (in Chinese). (肖啸菴, 汪德熙, 核化学与放射化学, 1991, 13, 1.)

[5] Lewis, G. N.; Macdonald, R. T. J. Am. Chem. Soc. 1936, 58, 2519.

[6] Brooks, S. C.; Southworth, G. R. Environ. Pollut. 2011, 159, 219.

[7] Pedersen, C. J. J. Am. Chem. Soc. 1967, 89, 7017.

[8] Dietrich, B.; Lehn, J. M.; Sauvage, J. P. Tetrahedron Lett. 1969, 10, 2885.

[9] Alexandratos, S. D.; Stine, C. L. React. Funct. Polym. 2004, 60, 3.

[10] Gokel, G. W.; Leevy, W. M.; Weber, M. E. Chem. Rev. 2004, 104, 2723.

[11] Gokel, G. W.; Daschbach, M. M. Coord. Chem. Rev. 2008, 252, 886.

[12] Olsher, U.; Izatt, R. M.; Bradshaw, J. S.; Dalley, N. K. Chem. Rev. 1991, 91, 137.

[13] Shirai, A.; Ikeda, Y. Inorg. Chem. 2011, 50, 1619.

[14] Jepson, B. E.; Carins, G. A. Report MLM-2622, 1979.

[15] Chen, Y.-H. Chin. J. Rare Met. 1983, 79 (in Chinese). (陈耀焕，稀有金属, 1983, 79.)

[16] Li, T.-W. At. Energy Sci. Technol. 1990, 24, 87 (in Chinese). (李廷伍, 原子能科学与技术, 1990, 24, 87.)

[17] Gu, Z.-G.; Li, Z.-J.; Yang, J. Prog. Chem. 2011, 23, 1892 (in Chinese). (顾志国, 李在均, 杨杰, 化学进展, 2011, 23, 1892.)

[18] Zhi, K.-Z.; Dou, F.-Q.; Yang, K.-S. At. Energy Sci. Technol. 1982, 16, 686 (in Chinese). (支克正，窦富全，杨坤山，原子能科学技术, 1982, 16, 686.)

[19] Zhi, K.-Z.; Dou, F.-Q.; Yang, K.-S. At. Energy Sci. Technol. 1983, 17, 347 (in Chinese).

(支克正, 窦富全, 杨坤山, 原子能科学技术, 1983, 17, 347.)
[20] Fang, S.-Q.; Fu, L.-A. J. Nucl. Radiochem. 1984, 6, 40 (in Chinese). (方胜强, 傅立安, 核化学与放射化学, 1984, 6, 40.)

[21] Fang, S.-Q.; Zhi, K.-Z.; Fu, L.-A. J. Nucl. Radiochem. 1987, 9, 142 (in Chinese). (方胜强, 支克正, 傅立安, 核化学与放射化学, 1987, 9, 142.)

[22] Chen, Y.-H.; Sheng, H.-Y. At. Energy Sci. Technol. 1985, 19, 595 (in Chinese). (陈耀焕，盛怀禹，原子能科学技术, 1985, 19, 595.)

[23] Jiang, Y.-L.; Zhang, X.-X.; Qian, J.-H. At. Energy Sci. Technol. 1986, 20, 2 (in Chinese). (姜延林, 张心祥, 钱建华, 原子能科学技术, 1986, 20, 2.)

[24] Fu, L.-A.; Fang, S.-Q.; Zhang, F.-S. J. Nucl. Radiochem. 1989, 11, 142 (in Chinese). (傅立安, 方胜强, 张复昇, 核化学与放射化学, 1989, 11, 142.)

[25] Fang, S.-Q.; Fu, L.-A. J. Nucl. Radiochem. 1991, 13, 87 (in Chinese). (方胜强, 傅立安, 核化学与放射化学, 1991, 13, 87.)

[26] Fang, S.-Q.; Fu, L.-A.; Gao, Z.-C. J. Nucl. Radiochem. 1992, 14, 111 (in Chinese). (方胜强, 傅立安, 高志昌, 核化学与放射化学, 1992, 14, 111.)

[27] Fang, S.-Q.; Fu, L.-A. J. Isotopes 1994, 7, 168 (in Chinese). (方胜强, 傅立安, 同位素, 1994, 7, 168.)

[28] Jin, J.-N.; Wang, Q.-J.; Meng, M.-L. J. Sichuan Univ. 1999, 36, 903 (in Chinese) (金建南, 王全基, 孟明礼, 四川大学学报, 1999, 36, 903.)

[29] Fujine, S.; Saito, K.; Shiba, K. J. Nucl. Sci. Technol. 1983, 20, 439.

[30] Nishizawa, K.; Watanabe, H.; Ishino, S.; Shinagawa, M. J. Nucl. Sci. Technol. 1984, 21, 133.

[31] Kim, D. W.; Jeon, Y. S.; Eom, T. Y.; Suh, M. Y.; Lee, C. H. J. Radioanal. Nucl. Chem. 1991, 150, 417.

[32] Kim, D. W.; Jeon, Y. S.; Jeong, Y. K.; Suh, M. Y.; Joe, K. S. J. Radioanal. Nucl. Chem. 1995, 189, 219.

[33] Kim, D. W.; Hong, C. P.; Kim, C. S.; Jeong, Y. K.; Jeon, Y. S.; Lee, J. K. J. Radioanal. Nucl. Chem. 1997, 220, 229.

[34] Kim, D. W.; Jang, Y. H.; Lee, N. S.; Chung, Y. S.; Kim, K. Y.; Park, S. U.; Kim, C. S. J. Radioanal. Nucl. Chem. 1999, 240, 155.

[35] Kim, D. W.; Kim, C. S.; Jeon, J. S.; Kim, J. S.; Lee, N. S. J. Radioanal. Nucl. Chem. 1999, 241, 379.

[36] Kim, D. W.; Kim, B. K.; Park, S. R.; Lee, N. S.; Jeon, Y. S.; Choi, K. Y.; Lee, Y. I. J. Radioanal. Nucl. Chem. 1998, 232, 257.

[37] Kim, D. W.; Park, H. K.; Kim, C. S.; Jeon, Y. S. J. Radioanal. Nucl. Chem. 1999, 242, 769.

[38] Kim, D. W. J. Radioanal. Nucl. Chem. 2002, 253, 67.

[39] Kim, D. W.; Kim, C. S.; Lee, N. S.; Ryuc, H.; Kim, J. S.; Jang, Y. H. Naturforscher 2002, 57, 107.

[40] Kim, D. W.; Park, S. R.; Kim, S. J.; Kim, H. J. J. Radioanal. Nucl. Chem. 1998, 229, 165.

[41] Kim, D. W.; Kim, H. J.; Kim, M. S.; Choi, K. Y.; Kwon, S. H.; Shin, J. S.; Kim, J. S. J. Radioanal. Nucl. Chem. 2001, 247, 385.

[42] Kim, D. W.; Kim, J. S.; Jeon, J. S.; Kim, H. J.; Lee, N. S. J. Radioanal. Nucl. Chem. 1999, 242, 215.

[43] Kim, D. W.; Kim, H. J.; Jeon, J. S.; Choi, K. Y.; Jeon, Y. S. J. Radioanal. Nucl. Chem. 2000, 245, 571.

[44] Kim, D. W.; Kang, B. M.; Jeon, B. K.; Jeon, Y. S. J. Radioanal. Nucl. Chem. 2003, 256, 81 .

[45] Ban, Y.; Nomura, M.; Fujii, Y. J. Nucl. Sci. Technol. 2002, 39, 279.

[46] Otake, K.; Suzuki, T.; Kim, H. J.; Nomura, M.; Fujji, Y. J. Nucl. Sci. Technol. 2006, 43, 419.

[47] Jeon, Y. S.; Jang, N. H.; Kang, B. M.; Jeon, Y. S.; Kim, C. S.; Choi, K. M.; Ryu, H. Bull. Korean Chem. Soc. 2007, $28,451$.

[48] Lee, D. A. J. Inorg. Nucl. Chem. 1976, 38, 161. 
[49] Mezei, G.; Zaleski, C. M.; Pecoraro, V. L. Chem. Rev. 2007, 107, 4933.

[50] Piotrowski, H.; Polborn, K.; Hilt, G.; Severin, K. J. Am. Chem. Soc. 2001, 123, 2699.

[51] Piotrowski, H.; Hilt, G.; Schulz, A.; Mayer, P.; Polborn, K.; Severin, K. Chem. Eur. J. 2001, 7, 3196.

[52] Severin, K. Coord. Chem. Rev. 2003, 245, 3.

[53] Grote, Z.; Lehaire, M.-L.; Scopelliti, R.; Severin, K. J. Am. Chem. Soc. 2003, 125, 13638.

[54] Grote, Z.; Scopelliti, R.; Severin, K. J. Am. Chem. Soc. 2004, 126, 16959.

[55] Grote, Z.; Wizemann, H. D.; Scopelliti, R.; Severin, K. Z. Anorg. Allg. Chem. 2007, 633, 858 .
[56] Katsuta, S.; Iwabe, Y.; Kato, Y.; Kudo, Y.; Takeda, Y. Inorg. Chim. Acta 2008, 361, 103.

[57] Graf, E.; Hosseini, M. W. Coord. Chem. Rev. 1998, 178 180, 1193.

[58] Abe, A. M. M.; Helaja, J.; Koskinen, A. M. P. Org. Lett. 2006, 8, 4537.

[59] Arens, V. A.; Dietz, C.; Schollmeyer, D.; Jurkschat, K. Organometallics 2013, 32, 2775.

[60] Wendji, A. S.; Lutter, M.; Dietz, C.; Jouikov, V.; Jurkschat, K. Organometallics 2013, 32, 5720.

[61] Boda, A.; Ali, S. M.; Rao, H.; Ghosh, S. K. J. Mol. Model. 2012, 18,3507 . 